Title: Understanding how cancer patients actualise, relinquish, and reject advance care planning: Implications for practice

Short Title: Advance care planning with cancer patients

First and Corresponding Author:

Dr Natasha Michael, Consultant Physician in Palliative Medicine. Department of Pain and Palliative

Care, Peter MacCallum Cancer Centre, Peter Mac, Locked Bag 1, A'Beckett Street, Victoria, 8006, Australia.

Phone: +61396561918 Fax: +61396567327

Email: natasha.michael@petermac.org

Other Authors:

Dr Clare O’Callaghan. Department of Pain and Palliative Care, Peter MacCallum Cancer Centre; Departments of Oncology and Medicine, The University of Melbourne, Australia.

A/Prof Josephine Clayton. HammondCare Palliative and Supportive Care Service \& University of Sydney, Australia

Dr Annabel Pollard. Department of Psychology, Peter MacCallum Cancer Centre, Melbourne, Australia

Nikola Stepanov. School of Population Health \& Melbourne Medical School, The University of Melbourne, Australia

Dr Odette Spruyt. Department of Pain and Palliative Care, Peter MacCallum Cancer Centre, Melbourne, Australia.

A/Prof Michael Michael. Department of Cancer Medicine, Peter MacCallum Cancer Centre, Melbourne, Australia.

Prof. David Ball. Department of Radiation Oncology, Peter MacCallum Cancer Centre, Melbourne, Australia. 


\begin{abstract}
Purpose Although advance care planning (ACP) is recognised as integral to quality cancer care, it remains poorly integrated in many settings. Given cancer patients' unpredictable disease trajectories and equivocal treatment options, a disease specific ACP model may be necessary. This study examines how Australian cancer patients consider ACP. Responses will inform the development of an Australian Cancer Centre's ACP programme.

Methods A constructivist research approach with grounded theory design was applied. Eighteen adults from lung and gastro-intestinal tumour streams participated. Participants first described their initial understanding of ACP, received ACP information, and finally completed a semi-structured interview assisted by the vignette technique. Qualitative inter-rater reliability was integrated. Results Participants initially had scant knowledge of ACP. On obtaining further information, their responses indicated that: For cancer patients, ACP is an individualised, dynamic, and shared process characterised by myriad variations in choices to actualise, relinquish, and/or reject its individual components (medical enduring power of attorney, statement of choices, refusal of treatment certificate, and advanced directive). Actualisation of each component involves considering, possibly conversing about, planning, and communicating a decision, usually iteratively. Reactions can change over time and are informed by values, memories, personalities, health perceptions, appreciation of prognoses, and trust or doubts in their substitute decision makers.
\end{abstract}

Conclusion Findings endorse the value of routinely, though sensitively discussing ACP with cancer patients at various times points across their disease trajectory. Nonetheless, ACP may also be relinquished or rejected and ongoing offers for ACP in some patients may be offensive to their value system.

Keywords: advance care planning; cancer; adults; advance directives 


\section{Understanding how cancer patients actualise, relinquish, and reject advance care planning:}

\section{Implications for practice}

\section{Background}

Advance Care Planning (ACP) is a process of reflection and communication in which a capable person makes decisions related to future health and/or personal care in the event that they become incapable of giving informed consent [1]. Advance Care planning can take many forms and in the state of Victoria, Australia, may include the following components: Medical Enduring Power of Attorney (MEPOA) ${ }^{1}$, Statement of Choices ${ }^{2}$, Refusal of Treatment Certificate ${ }^{3}$, and/or an Advanced Directive (AD) ${ }^{4}$.

Although ACP is recognised as integral to the provision of high quality cancer care $[4,5]$, it remains poorly incorporated into the routine care of cancer patients. In a study of American patients with incurable pancreatic cancers, only $15 \%$ of 1186 people had ADs in their medical records [6]. Furthermore, a retrospective analysis exploring completion of "do not resuscitate" (DNR) directives of 206,437 American cancer patients found that $63 \%$ of the inpatient adults with capacity (53\% of DNR deaths) and surrogates of $95 \%$ of those who lacked capacity (34\% of DNR deaths) signed DNR orders on the day of death [7].

In Australia, a single randomised control study of 309 participants confirmed the efficacy of ACP in elderly inpatients with various diagnoses during a hospital admission [8]. Compared to standard care, patients who received a facilitated ACP intervention were more likely to have end-of-life preferences known and respected (86\% intervention group patients who died versus $31 \%$ in the control group, $\mathrm{p}<0.001)$, patients and family were more satisfied with care $(\mathrm{p}<0.001)$, and family members reported less anxiety $(\mathrm{p}=0.02)$ and depression $(\mathrm{p}=0.002)$ after patients' deaths. It is unclear whether these findings transfer to Australian cancer patients with a wider age range, unpredictable disease trajectories, and equivocal treatment options. Patients with advanced cancer vary in their willingness

\footnotetext{
${ }^{1}$ A MEPOA is a legal document where an individual (the 'donor') appoints another person (the 'agent') to make decisions about medical treatment on their behalf in circumstances when the donor becomes incompetent and is unable to make decisions for themselves due to accident or illness resulting in incapacity [2].

${ }^{2}$ A Statement of Choices provides specific information related to a person's wishes and values [3].

${ }^{3}$ A Refusal of Treatment Certificate applies to the refusal of a medical treatment for a current condition and not to an illness / condition that may occur in the future. It does not allow for the refusal of palliative care [2].

${ }^{4}$ An advance directive (AD) is typically defined as a document which is created by a person while they are competent, that defines the medical treatment that the person wishes to refuse should they become incompetent in the defined circumstances [2].
} 
and desire to engage in ACP discussions [9-11] and often refuse to participate in ACP research [12, 13]. Low ACP uptake in cancer patients may also relate to poor understanding of treatment intent: Some misinterpret palliative treatment as potentially curative [14] and have unrealistic survival expectations [13].

A qualitative study of 22 cancer patients in the United Kingdom highlighted that, cancer patients commonly consider ACP but prefer discussions well into their illness trajectory, across multiple occasions, and when initiated by skilled professionals who treat them as individuals [12]. Whilst ACP enhances a sense of control [12], it can negatively affect patient-doctor relations [12], either annihilate $[12,13]$ or encourage hope [15], and should be reviewed on a regular basis [12]. In a study exploring the views of 25 lung cancer patients and 19 family members, patients wanted to "carry on as normal" and "maintain integrity", rather than focus on end-of-life care preferences [16].

The American Society of Clinical Oncology recommends oncologists initiate individualised ACP assessments throughout the illness course and not simply at end stages [17], offering regular reviews when health circumstances change. However, cancer patients may be reluctant to initiate ACP discussions with oncologists: "you go to the oncologist to be cured, not to be buried" [10]; may want physicians to kick-start conversations or prefer discussions with unfamiliar physicians, such as the admitting doctor [18]. Systematic reviews highlight that clinician-initiated [19] and multiple ACP discussions [20] lead to higher AD completion rates. Discussions should be timed to follow adjustment to the diagnosis [21], recognise the capacity to integrate hope with truthful communication [21, 22], and accommodate the preferences of those from culturally and linguistically diverse backgrounds [23]. Additionally, tailoring ACP programmes to the needs of cancer patients and their oncologists may lead to more meaningful and acceptable conversations, thus enabling consideration of individual cancer trajectories and treatment complexities $[24,25]$.

This study aimed to further an appreciation of how cancer patients consider ACP so that patients' responses could inform the development of an ACP programme in an Australian Cancer Centre.

\section{Methods}

This constructivist research draws on the Medical Research Council framework [26] for the development and evaluation of complex interventions. Constructivism focuses on the role of ideas, norms, knowledge, and culture in an individual's construction of social experiences [27]. The research 
approach used grounded theory [28] and the vignette technique. Vignettes are "short stories about characters in specified circumstances, to whose situation the interviewee is invited to respond" [29]. They are useful in exploring potentially sensitive topics [30], allow greater control over interactions, and enable participants to determine when and if they introduce personal experiences.

A research advisory group (oncologist, palliative care physician, ethicist, consumers, and clinical psychologist) supported development and piloting of research material (vignettes) and recruitment strategies. The four vignettes reflected patients at different cancer stages, with varying degrees of ill health, symptoms, and cognitive involvement (See Appendix 1). Ethics approval was gained for the study.

Participants

Participants were from the lung and gastro-intestinal tumour streams, over 25-years-old, sufficiently proficient in English and judged by clinicians as suitable for the study. Those with a $<4$ week prognosis, Eastern Cooperative Oncology Group score of 4, and cognitive impairment ( $\leq 22$ Mini Mental State Examination) were excluded. Background data was obtained from interviews and patients' files.

Procedure

Participants were approached by the Principal Investigator in person or by telephone. Sampling was theoretical [28] and extended to those thought able to share varied perspectives about ACP during March-August, 2012. Ongoing sampling occurred until findings were saturated. Stages following recruitment included: exploring initial understanding of ACP; providing information on $\mathrm{ACP}$; and meeting with researcher to read vignettes and complete a semi-structured interview (Table 1). Mean interview times were 69 (SD 14.3) minutes (range, 43 to 95 minutes).

Analysis

Analysis was cyclic and included: inductive coding; condensing comparable codes into researcher created categories; condensing comparable categories into themes; and creating a theoretical statement of findings. The second author transcribed interviews and conducted initial data analysis, assisted by data management software [31]. Three further authors provided qualitative inter-rater reliability [32] through reading interview transcripts and verifying or extending the analysis.

\section{Results}

Twenty-nine patients were approached, considered participation, and were given consent forms. Six 
“declined" when followed up: 5 were too unwell and one did not return calls. Five withdrew following discussion and receiving ACP information. Family (4) or medical staff (1) reported they were unwell or unavailable. Characteristics of 18 participants who gave informed consent and completed interviews are in Table 2. Some participants, who expressed occasional distress during interviews, were offered additional support.

Interview analysis yielded 953 codes and 13 categories. Coding and category development are illustrated in Tables 3 and 4. Table 4 also presents examples of participants' statements which informed categories. Comparable categories were condensed into three themes, denoted by A-C (Table 4). Participants also made suggestions for offering ACP to cancer patients which were summarised (Table $5)$.

Theme A: ACP remains poorly understood and individualised Initial exploration of participants' understanding about ACP indicated vague or no direct knowledge. Seven guessed or assumed it was planning for future illness stages and one defined it as outpatient follow-up.

After receiving further information, participants' reports suggested they varied considerably in the ACP components they had already conceptualised and completed. They expressed interest in diverse aspects including MEPOA, organ donor status, preparing memorials, eulogies, and planning for dependents' care. One participant differentiated health related ACP from personal "bucket list" plans. Some believed planning for their funeral would support their bereaved while others did not want to "dictate from the grave".

Participants also differed in views about tolerable levels of debility and acceptability of lifeprolonging treatment. Younger participants with dependent children considered more life-prolonging treatments (see Table 4, A4). Others did not want life prolonged if they lost consciousness, the ability to communicate, had uncontrolled pain, burdened family or society, or had scant hope of improvement. One 60-year-old female ${ }^{5}$, however, said that her daughter lived abroad and added, "I would like to wait for her to come and (for doctors to) prolong my life but if she was here then of course I prefer not to extend my life artificially."

\footnotetext{
${ }^{5}$ Ages and genders are stated to indicate the different participants (there were no patients with same age and gender).
} 
Some participants were already recipients of palliative care services whilst others questioned what it involved. Many expressed a desire for death at home, yet others were open to inpatient care. One young mother planned hospice care to maintain dignity, concerned about burdening her family with care needs. Others were aware of potential end-of-life challenges but not ready, wanting, or able to make related decisions, even when living with advanced illness, possibly because the potential illness course was unknown.

Theme B: ACP is dynamic and shared ACP is a dynamic process of actualising, relinquishing, and /or rejecting its specific components (Fig1 Patients reactions to ACP). Actualisation means that participants may: conceptualise an ACP component, consider and possibly talk to others about it, plan, and communicate decisions. Alternately, some reject ${ }^{6}$ or delay ACP, or decide on one or two components and not consider or relinquish ${ }^{7}$ others. $^{2}$ A 67-year-old male explained that, "it's up to a doctor to decide whether the medical outlook is that you can be resuscitated". ACP could also be totally relinquished. One 58-year-old Chinese male stated: "I haven't thought about (ACP) because naturally (children) ... take care of the parents ... that's what they are bringed up with". Over two thirds of participants suggested that they would want to be able to change ACP decisions.

Participants welcomed ACP as part of routine care but often wanted to consider different ACP components at various times. A 49-year-old female currently organising end-of-life care said that she did not want to consider ACP when initially diagnosed stating, "It's not something you'd want to dwell on while you're enjoying the day to ... think about cancer, cancer, cancer all the time". Despite having appointed a MEPOA, some wanted to delay treatment and end-of-life care decisions until their conditions became more advanced. Sensitivity to family or friends' reactions was important when timing conversations, as a 63-year-old male reported:

'My friend (MEPOA) is also very optimistic ... I'm probably a bit more kind of realistic ... it's that balancing of not wanting to sort of pop the balloon of optimism ... by focussing too much on what can go wrong.'

Many described ACP as an iterative process as they considered their priorities, values, and futures. The 63-year-old male said that thoughts about his funeral, "come to the fore and to the back of

\footnotetext{
${ }^{6}$ To refuse to engage in all or some of ACP components and decisions.

${ }^{7}$ To consciously hand over ACP decisions and / or have an expectation that others will assume ACP decisions on their behalf
} 
your mind as you think of the remote chance (for remission)" and described his fear and hope as a "competition" and "contradiction". Contradictory remarks were also made with regards to health and ACP decisions (Table 4, B2). A well-educated 67-year-old patient said that he had.

'some cells, ... in one vertebrae and the liver and a couple of cells somewhere in the head. (later) What am I going to do if ... the cancer is spread everywhere and it's in my brain?" (later) I'm not terribly worried about my cancer, what does worry me is the possibility of getting some other disease ... that will make me seriously ill or kill me, ... or even another cancer.'

Participants discussed consulting, sharing, and informing others about their ACP decisions in varied ways, with conversations being spontaneous or planned, and decisions verbalised or written. A number described how they were happy to discuss different components with different people and many favoured ACP discussions with someone specifically trained. Two stated that they hadn't intended discussing ACP with family or health professionals but the interview had changed their mind. Theme C. Biopsychosocial and metaphysical informants of ACP Many participants were not religious, and only occasionally specified how spiritual and cultural backgrounds affected their ACP. One participant's Scottish heritage prompted her request for mirror coverings upon death, and another associated Protestantism with desiring a low cost funeral. Two perceived that their ACP decisions contradicted their backgrounds: a Christian did not want life extended "at all costs" and a Muslim wanted cremation rather than burial. A Buddhist was also not concerned about what happened after her death. Alternately, a Christian emphasised that religion was fundamental to his request that treatment not shorten his lifespan (Table 4, Category C1).

Participants' backgrounds prior to and since diagnoses influence ACP. Positive memories of a mother's end-of-life care triggered one participant's plan to request palliative care but uncomfortable memories of family members', friends', and acquaintances' advanced illnesses and comparable media reports also resulted in other participants not wanting to consider life-extending treatment when seriously unwell.

Most considered families' and/or friends' welfare when considering ACP. Concerns were expressed about physically or financially burdening family, and "wasting" health resources if unlikely 
to improve. Others wanted life prolonged to remain alive with young family, even if this was "semiselfish" (Table 3).

Personality, including adjustment and coping styles may affect decisions, their timing, and accompanying emotional reactions. Staying positive and "not giving up" was sometimes associated with delaying ACP or planning to not think about death until the "time comes". These patients, nonetheless, may also make fleeting comments to family members about their wishes. A self-described "realist" said that if her illness progressed she would hope for the best but plan for the worst. "Laziness", distractibility, and fatalism were also mentioned as reasons for not proceeding with ACP. Participants also considered life prolonging treatment when wanting to reach personal milestones, such as a $50^{\text {th }}$ birthday, and two were concerned that communicating ACP decisions would threaten cancer treatment.

Approaching specific ACP components can be difficult, distressing, and/or helpful. A 59year-old male stated:

(I want) to be cremated and preferably if the ashes could be spread ... beyond the bay

... classical music ... it's a fantasy ... (which) nurtures the values that I have ... the essence of life that I look forward to. ... The very thought is always very nourishing.

Illness uncertainty either triggered participants' ACP or justified its delay. Some excused their delay of ACP by commenting on their perceived good health and prognoses, including the 67-year-old male mentioned earlier who described his metastatic illness and made contradictory statements about neural involvement.

While most believed that their informal and professional carers would enact their wishes, occasional doubts were expressed (Table 4, Category C6). The 63-year-old male was "anxious" about a possible "gap" between desired end-of-life care and "the value judgement of a health care professional about what they can do". Some also wanted family to make their life-prolonging treatment decisions in consultation with health professionals (Table 3).

Given the saturated findings, a "substantive grounded theory" [28] is proposed to explain how a cohort of Australian patients living with life-threatening cancer respond to ACP: ACP is an individualised, yet dynamic and shared process in cancer patients' minds, discussions, and actions. It is characterised by variations in actualising, relinquishing, and/or rejecting its individual components. 
The actualisation of each ACP component involves considering, possibly conversing about, planning, and communicating a decision usually in an iterative manner. Reactions to ACP are informed by: family, community, and metaphysical values; memories; personalities; health perceptions, and trust or doubts in their family and broader social and health care networks. Individual approaches to ACP, related decisions, or avoidance of ACP can change over time.

\section{Discussion and implications for practice}

These results suggest that Australian patients living with incurable cancer may respond to ACP in myriad ways (Table 5). It supports previous findings that cancer patients widely vary in wanting to engage in $\mathrm{ACP}[9,12,16]$ but may welcome discussions $[33,34]$. If poorly timed, the experience may reduce personal integrity [16] and positivity [9]. Participants occasionally doubt that their wishes will be enacted [33] but trust medical carers to make decisions on their behalf if needed [9]. Cancer patients often find it difficult to make decisions without adequate knowledge of their likely prognosis, the process of deterioration, options for place of care, and future healthcare choices [9]. For some, decisions are also affected by memories of illness and concerns for the welfare of their family $[9,16]$. Patients also often want to consider different ACP components at different points in their illness trajectory rather than as a single event or a series of closely timed discussions, as can occur in ACP research [8]. Despite occasional distress, many outlined the interview’s therapeutic benefit.

Our findings illustrate that patients require time and support in conceptualising ACP. Some continued to display a poor understanding of ACP, despite having been provided with written information prior to the interviews; suggesting that patients may require detailed verbal clarification of their understanding prior to and even following completion of discussions or documentation. These findings may imply poor overall general public awareness on ACP and have significant clinical implications considering widespread national end of life initiatives that promote ACP. Thus the current emphasis on ACP for patients with advance illness within healthcare settings may be self limiting in its effect by delaying the introduction of this necessary but sensitive topic for consideration when patients are most unwell.

Participants suggested that ACP should be promoted as part of "routine care" offered to all cancer patients (Table 5), and not introduced after significant health events, so as not to "scare patients". This approach may address one of the main barriers to ACP, that is, an invitation to complete ACP signifies a limited prognosis or significant risk of health deterioration [34]. ACP programmes 
should offer easily accessible and varied information and educational materials to enable patients to decide when and if they would like to proceed with discussing various components of ACP. Patients may also sometimes want tailored advice in completing advance care plans including, which option is most appropriate for their circumstances at a particular time.

Participants' views importantly illustrate how some patients may choose to not to discuss ACP or even relinquish decisions to others. In such cases, providing ongoing opportunities to consider ACP [16] could be viewed as insensitive and contrary to the patient's autonomous choice [35]. Such patients are arguably making a self-determining "choice to not have to choose" [36], possibly expressing an identity that is rooted in family and social collectivist affinities [35] or a coping/adjustment style of "trying to keep positive". When this occurs, practitioners must be mindful that patients and/or families still trust their opinions and may choose to rely on their expertise in the future to guide patient care and determine best practice [37].

Some participants viewed their prognosis more optimistically than was likely realistic and made incorrect assumptions of the intent of their treatment. These may have been used to justify delaying ACP. A recent study of a cohort of 1193 patients with Stage IV lung or colorectal cancer found that, paradoxically, patients who reported higher scores for physician communication were at greater risk for inaccurate treatment expectations [38]. Findings also suggested that patients may perceive physicians as better communicators when they convey a more optimistic view of treatment. Cancer patients who overestimate survival are also more likely to seek life extending therapy over comfort care [39] and their estimation may be influenced by their values, understanding, or even denial of illness [40].

Whilst cancer patients remain experts on their own preferences, the oncologist likely remains the authority on the possible illness course and treatment options [41]. Physicians, though, have been shown to lack skills in communicating prognosis and conducting ACP conversations [42]. A number of strategies to improve engagement, including competency training, the use of prompts, and checklists to assist with discussions have been recommended [41, 43]. Nevertheless, successfully engaging cancer patients in ACP is most likely to be influenced by whether discussions incorporate what is meaningful in patients' lives $[9,16]$ and recognise patients' need for significance, belonging, and connection with others when they are dealing with stressful diagnoses [44]. 
Finally, this study reinforces findings on the dynamic process of decision-making in $\mathrm{ACP}$, as participants described the need to change decisions over time, or made contradictory statements regarding their perceived health statuses and ACP decisions within interviews. Sudore et al. recommends that ACP be considered as a cyclic process which includes phases of contemplation, preparation, action, and maintenance [45]. We suggest that ACP is more of an iterative than cyclic process and includes options of relinquishing or rejecting decisions about its individual components. The oscillations noted as participants avoided or approached ACP also require further consideration. Patients with advanced cancer identify loss and grief as central issues [46] and, in contemplating ACP, cancer patients may grieve for their once healthy selves, family systems, and future hopes.

Nonetheless, Robinson found that cancer patients can hope for a possible cure and more good times whilst still planning for end-of-life, as this helps them to keep living well [15]. The apparent inconsistent reactions in this study may similarly reflect participants' efforts to continue to live a quality life. Sensitive accommodation of frequent contradictions is therefore needed in ACP interventions.

\section{Limitations and future research}

The small sample size and omission of non-English speaking and young participants excluded patients with additional insights. Whilst the vignette technique was helpful for most patients who found them personally relevant, one did not think they were appropriate to her cancer experience, one could not recall them, and another thought that they could be frightening. These findings and an evaluation of cancer patients' carers' views on ACP (ongoing) will inform an ACP intervention that will be examined in a Phase II feasibility study.

\section{Conclusion}

This study extends our understanding of how cancer patients consider ACP through illustrating the dynamic, individualised nature of actualising, relinquishing, or rejecting decisions over time, privately or with others. Participants endorsed routine discussions on ACP through individual negotiation to discern information needs and involvement preferences. ACP may still be acceptable to patients who initially reject it if the approach is reframed and incorporates key elements of sensitive communication as occurred in these guided interviews. However criteria for successful outcomes should include the choice not to discuss future care plans, recognising that offering ongoing ACP opportunities may be offensive to the value system of some patients. 


\section{Acknowledgements}

The authors thank A/ Prof Mei Krishnasamy for her comments on the manuscript, staff from the lung and gastrointestinal tumour streams and research staff for their support in this project. We are grateful to patients who generously participated. NM was responsible for study concept and secured funding. NM, AP, OS, JC, and NS were responsible for study design. NM and COC liaised with the consultant oncologists, recruited and gained written informed consent from the patients, collected the data, and managed the trial on a daily basis. BD and MM facilitated recruitment of patients. NM, COC, AP, and NS performed analysis and interpretation of results. NM and COC produced first draft of manuscript. AP. OS, NS, and JC contributed to subsequent revisions. Critical revision of the manuscript for important intellectual content was done by all authors. Final approval of the article was done by all authors. NM is the guarantor. This research was supported by a grant from the Department of Health, Victoria. The research team were independent from the funders.

\section{Conflict of interest}

The authors declare no conflict of interest. NM and COC have full control of all primary data and will allow review of data if requested

\section{References}

1. Advance care planning in Canada: A national framework and implementation. National roundtable proceedings. 2009, Canadian Hospice Palliative Care Association

2. Office of the Public Adocate (2012) Promoting the rights, interests and dignity of people with a disability. Victoria, Australia. http://www.publicadvocate.vic.gov.au/. Accessed 6 October 2012

3. Respecting Patient Choices (2012) http://www.respectingpatientchoices.org.au/. Accessed 6 October 2012

4. Walling A, Lorenz KA, Dy SM, Naeim A, Sanati H, Asch, SM, Wenger NS (2008) Evidencebased recommendations for information and care planning in cancer care. J Clin Oncol 26:3896-3902 
5. Epstein AS, Volandes AE, O'Reilly EM (2011) Building on individual, state, and federal initiatives for advance care planning, an integral component of palliative and end-of-life cancer care. J Oncol Pract 7(6):355-359

6. Tan TS, Jatoi A (2008) An update on advance directives in the medical record: findings from 1186 consecutive patients with unresectable exocrine pancreas cancer. J Gastrointest Cancer, $200839: 100-3$

7. Levin TT, Li Y, Weiner JS, Lewis F, Bartell A, Piercy J, Kissane DW (2008) How do-notresuscitate orders are utilized in cancer patients: timing relative to death and communicationtraining implications. Palliat Support Care 6(4):341-8

8. Detering KM, Hancock, AD, Reade MC, Silvester W (2010) The impact of advance care planning on end of life care in elderly patients: randomised controlled trial. BMJ. doi:10.1136/bmj.c1345

9. Barnes KA, Barlow CA, Harrington J, Ornadel K, Tookman A, King M, Jones L (2011) Advance care planning discussions in advanced cancer: analysis of dialogues between patients and care planning mediators. Palliat Support Care 9(1):73-9

10. Lamont EB, Siegler M (2000) Paradoxes in cancer patients' advance care planning. J Palliat Med 3:27-35

11. Jones L, Harrington J, Barlow CA, Tookman A, Drake R, and Barnes K, King M (2011) Advancecare planning in advanced cancer: can it be achieved? An exploratory randomized patient preference trial of a care planning discussion. Palliat Support Care 9:3-13

12. Barnes K, Jones L, Tookman A, King M (2007) Acceptability of an advance care planning interview schedule: a focus group study. Palliat Med 21:23-8

13. Fu S, Barber FD, Naing A, Wheler J, Hong D, Falchook G, et al (2012) Advance care planning in patients with cancer referred to a phase I clinical trials program: the MD Anderson Cancer Center experience. J Clin Oncol 30:2891-6

14. Gattellari M, Butow PN, Tattersall MH, Dunn SM, MacLeod CA (1999) Misunderstanding in cancer patients: why shoot the messenger? Ann Oncol 10:39-46

15. Robinson CA (2012) "Our best hope is a cure." Hope in the context of advance care planning. Palliat Support Care 10:1-8 
16. Horne G, Seymour J, Payne S (2012) Maintaining integrity in the face of death: A grounded theory to explain the perspectives of people affected by lung cancer about the expression of wishes for end of life care. Internat J Nurs Stud 49:718-726

17. Peppercorn JM, Smith TJ, Helft HR, DeBono DJ, Berry SR, Wollins DS et al (2011) American Society of Clinical Oncology Statement: Toward individualized care for patients with advanced cancer. J Clin Oncol 29:755-760

18. Dow LA, Matsuyama RK, Ramakrishnan V, Kuhn L, Lamont EB, Lyckholm L, Smith TJ (2010) Paradoxes in advance care planning: the complex relationship of oncology patients, their physicians, and advance medical directives. J Clin Oncol 28:299-304

19. Patel RV, Sinuff T, Cook DJ (2004) Influencing advance directive completion rates in nonterminally ill patients: a systematic review. J Crit Care 19:1-9

20. Hanson LC, Tulsky JA, Danis M (1997) Can clinical interventions change care at the end of life? Ann Intern Med 126:381-8

21. Ho A (2008) Relational autonomy or undue pressure? Family's role in medical decisionmaking. Scand J Caring Sci 22(1):128-135

22. Clayton JM, Butow PN, Arnold RM, Tattersall MH (2005) Discussing end-of-life issues with terminally ill cancer patients and their carers: a qualitative study. Support Care Cancer $13: 589-599$

23. Butow P, Sze M, Dugal-Beri P, Mikhail M, Eisenbruch M, Jefford, M et al (2011) From inside the bubble: migrants' perceptions of communication with the cancer team. Support Care Cancer 19:281-290

24. Bravo G, Dubois MF, Wagneur B (2008) Assessing the effectiveness of interventions to promote advance directives among older adults: A systematic review and multi-level analysis. Soc Sci Med 67:1122-1132

25. Berry SR, Singer PA (1998) The cancer specific advance directive. Cancer 82: 1570-1577

26. Craig P, Dieppe P, Macintyre S, Michie S, Nazareth I, Petticrew M (2006) Developing and evaluating complex interventions: new guidance. Medical Research Council. www.mrc.ac.uk/complexinterventionsguidance. Accessed 6 October 2012

27. Finnemore M, Sikkink (2001) TAKING STOCK: The constructivist research program in international relations and comparative politics. Annu Rev Polit Sci 4:301-416 
28. Corbin J, Strauss A (2008) Basics of qualitative research 3e: Techniques and procedures for developing grounded theory. Sage Publications, Thousand Oaks, California

29. Finch J (1987) The vignette technique in survey research. Sociology 21:105-114

30. Fins JJ, Maltby BS, Friedmann E, Greene MG, Norris K, Adelman R, Byock I (2005) Contracts, covenants and advance care planning: an empirical study of the moral obligations of patient and proxy. J Pain Symptom Manage 29:55-68

31. ATLAS V.5.5.9 (2009) ATLAS.ti Scientific Software Development, Berlin

32. Kitto SC, Chesters J, Grbich C (2008) Criteria for authors in the submission and assessment of qualitative research articles for the Medical Journal of Australia. Med J Aust 188:243-246

33. Horne G, Seymour J, Shepherd K (2006) Advance care planning for patients with inoperable lung cancer. Int J Palliat Nurs 12:172-8

34. Simon J, Murray A, Raffin S (2008) Facilitated advance care planning: what is the patient experience? J Palliat Care 24:256-264

35. Johnstone MJ, Kanitsaki O (2009) Ethics and advance care planning in a culturally diverse society. J Transcult Nurs 20:405-16

36. Candib L (2002) Truth telling and advance planning at the end of life: Problems with autonomy in a multicultural world. Fam Sys Health 20:213-228

37. Gillam L, Sullivan, J (2011) Ethics at the end of life: who should make decisions about treatment limitations for young children with life-threatening or life-limiting conditions? J Paeditr Child Health 47:594-598

38. Weeks JC, Catalano PJ, Cronin A, Finkelman MD, Mack JW, Keating NL, et al (2012) Pateints' expectations about effects of chemotherapy for advanced cancer. N Engl J Med 367:1616-1625.

39. Weeks JC, Cook F, O'Day SJ, Peterson LM, Wenger N, Reding D, et al (1998) Relationship between cancer patients' predictions of prognosis and their treatment preferences. JAMA 279:1709-1214

40. Zimmermann C (2004) Denial of impending death: a discourse analysis of the palliative care literature. Soc Sci Med 59:1769-1780

41. Billings JA (2012) The need for safeguards in advance care planning. JGIM 27:595-600 
42. Tulsky JA, Fischer GS, Rose MR, Arnold RM (1998) Opening the black box: how do physicians communicate about advance directives? Ann Intern Med 129:441-9

43. Clayton JM, Butow PN, Tattersall MH, Devine RJ, Simpson J M, Aggarwal G, et al (2007) Randomized controlled trial of a prompt list to help advanced cancer patients and their caregivers to ask questions about prognosis and end-of-life care. J Clin Oncol 25:715-23

44. Lethborg C, Aranda, S, Kissane D (2008) Meaning in adjustment to cancer: a model of care. Palliat Support Care 6:61-70

45. Sudore RL, Schickedanz AD, Landefeld CS, Williams BA, Lindquist K, Pantilat SZ, Schillinger D (2008) Engagement in multiple steps of the advance care planning process: a descriptive study of diverse older adults. J Am Geriatr Soc 56:1006-1013

46. Schofield P, Carey M, Love, A, Nehill C, Wein S (2006) 'Would you like to talk about your future treatment options?' discussing the transition from curative cancer treatment to palliative care. Palliat Med 20:397-406 
Table 1 Interview guide*

Q1. Do you identify your own current situation with any one of the scenarios?

Q2. At what point of your illness do you think you might want to have conversations about ACP?

Q3. What areas of ACP are you particularly interested in and why?

Q4. Some ACP documents ask patients to make decisions on starting or stopping treatments such as resuscitation, ventilation, antibiotics, and artificial feeding. How do you feel about making decisions about these issues at this stage? At another stage?

Q5. What influences the choices you may make about decisions related to care at the end of your life?

Q6. Having conversations on ACP can occur with your GP, treating doctor like ..., or somebody who is trained. Who would you feel most comfortable having these conversations with?

Q7. Do you think that you may change your mind about some of the decisions that you make in relation to your ACP over time?

Q6. Do you think an ACP should contain any other specific contents / what sort of other issues do you think you may want to document on your ACP?

Q7. Is there anything about completing an ACP that concerns you?

Q8. What do you think of this approach of using scenarios of cancer patients in different situations to introduce ACP to cancer patients?

Q9. Any other comments?

Q10. How has talking about ACP been for you?

Prompts and additional questions were tailored according to responses 
Table 2 Characteristics of Participants

Participants $(\mathrm{n}=18)$

Male: female

Mean Age

Inpatients: outpatients

Diagnoses:

Time Since Diagnosis:

Marital Status:

Education level:

Birthplace:

Place of interview
9 males: 9 females

59 yrs (SD 6; range 44-67 yrs)

2: 16

gastro-intestinal (10), lung (8)

$8(<1 \mathrm{yr}) \quad 7(1-5 \mathrm{yrs}) \quad 3(>5 \mathrm{yrs})$

7 single/separated/divorced

$10 \mathrm{married} / \mathrm{defacto}$

1 widowed

6 Some secondary

1 Finished secondary

4 Trade/college

5 Degree; 2 Masters/Phd

Australian (8); Taiwan/HK (2); Scotland (2); England (1);

USA (1); Poland (1); Holland (1); Spain (1); India (1)

Hospital interview room (10); participant's home (6) 
Table 3 Illustration of textual coding and category development (Categories listed in Table 4)

Text

Codes

Interviewer (I): ... resuscitation, ventilation, antibiotics

and artificial feeding. How do you feel about making

decisions about these issues at this stage? ...

M: If I was at scenario 4 (end stage cancer), not 3

(advanced cancer), because $3 \mathrm{I}$ believe there is still lots of hope even though its spread everywhere but you know miracle, miracles have happened you know there's some great treatment out there ... but scenario 4 if I was in that situation part of me would be saying I don't want to burden my family and put my family through all that and part of me would be saying I want to be there for my family so its semi-selfish. It's a very hard question to answer to be honest with you, ... I wouldn't want it to be a prolonged thing like months and months .... I would probably put my hands in the, and trust the, you know, my surgeon at the time, and, you know, whoever's dealing with me at the time to make those decisions, but I would like my wife to be involved as well. Although I don't want her to be in a position where she's making decision ... basically switch him off, on or off because I would hate her to have that decision because ... it'll eat her inside for If at scenario 1-3 would say prolong life as always hope (Informed Category A4)

If at scenario 4 hard to decide about prolonging measures: burden or being there for family (Informed Categories C3 \& C4)

Leave decisions to surgeon, carers \& wife (Informed Category B4)

Wants wife supported in decisions so doesn't eat away at her (Informed Category C3) many, many, years. 
Table 4 Textual examples (italics) informing categories depicting participants' perspectives about $\mathrm{ACP} *$

Categories

Text Illustrations

A1. Vague or no knowledge of ACP before and, sometimes, during the study.

\section{A2. Variations in needed ACP components}

A3. End-of-life care considered but seldom decided

A4. Varied tolerations of treatment and debility

B1. Actualising (conceptualising, considering, conversing, planning, and communicating), relinquishing, or rejecting decisions, and changing decisions, over time

B2. Oscillations and contradictions

B3. Informing about decisions in
I suppose I do (have an ACP). I go to Peter Mac every month and that's about it. ... Just for check-ups to see how I'm going (female, 46 yo).

No (doesn't need an MEPOA) I would just leave the family sort of thing, you know like let them do it (male, 64 yo**)

I want to die at home if possible but who's going to look after me? I need to get that all sorted (but will not make treatment decisions yet because) I feel I'm still going to have a life yet, for another good few years (female, 57 yo with advanced lung cancer)

I want everything possible to keep me going ... living off a machine or being in a wheelchair those things are nothing to me, I'd much rather be alive (male, 44 yo) I might change my mind (about ACP decisions) because we hang onto life because I think as long as I can feel I can have emotions I may decide to live, even if I suffered (female, 60 yo)

(After stating she did not want to live with advanced cancer) The reason I'm determined to try nearly every treatment, I want to see my ... grandchildren grow up, our daughter's getting married ..." (female, 63 yo) ... especially when there are emotional issues on the table, 
myriad ways C1. Spiritual and cultural factors: only
occasionally important

C2. Pre and post diagnosis memories affect ACP

C3. Family and community values

influence ACP

C4. Personality influences ACP

C5. Health perceptions affects ACP

C6. Trust and doubts in families, health carers, and institutional systems
... there's many opportunities for miscommunication so I

think the safest way to go is probably by documenting things (female, 53 yo)

I would not want my life cut off because I'm in so much pain for instance and you know I was still breathing by myself ... because my last breath is not up to anyone except the Lord (male, 59 yo)

She (mother) want to keep on going but I'm not sure I like that way to be artificial fed ... the injection ... because it's painful (female, 57 yo)

I'm going to be laying there as a cabbage and have the kids come in and I don't want this (male, 66 yo)

You wipe your bum and hold your doodle when you have a, have a piddle. That's when I don't want to go on, when I can't do that ... what's the point of going on? (male, 64 yo) I only have one lung now so ... I can get the flu and die ... I haven't many options ... and if it did come back ... liver or brain ... I definitely wouldn't have treatment, ... I know there's not a lot they can do (female, 58 yo) I want cremation ...(but) it might infringe the emotional ... values with the rest of the family but I don't want to impose, I just want to express my wishes (male, 59 yo)

\footnotetext{
* The three category groups informed three themes, A, B, C as listed in the Results.

$* *$ yo $=$ years old
} 
Table 5. Participants' suggestions for offering ACP to cancer patients

Introduction to ACP

Important not to scare people, e.g. if introduced after serious operation

ACP should be integrated as a routine "normal" process

Be gentle and gradual

Information provision

Offer information booklets, guides, DVDs, pocket sized materials for participants for when they need to know and when they are ready.

Do not overwhelm with too much reading material.

Give ACP documents to people to "sit on" and personally decide when to consider further. Information about the illness's possible course, possible treatments and their effects is needed to do ACP

ACP discussions

Different ACP components can be discussed with different people, e.g. legal matters with social workers.

Keep ACP staff consistent so that participants do not have to repeat things.

Only offer ACP if there are the staff resources available to assist; knowing someone is available for support if needed is also important.

When the patient does not want to talk about ACP, approach the patient through the family.

ACP can be done without help from the system:

I understand the system is being considerate in taking the wishes of a person but suppose we didn't have the conversations ... in my case I think I would still have related to my family as to what my wishes would be ... when I can't respond so those things (male, 59 years old)

Timing of Decisions

There is a different "right time" for introducing people ACP to different people. Contrasting times were suggested, including on diagnosis, not early after diagnosis, after one has "come to terms with the diagnosis", 6-12 months after diagnosis, before serious surgery, and "anytime". 
Figure 1 Patients' reactions to ACP

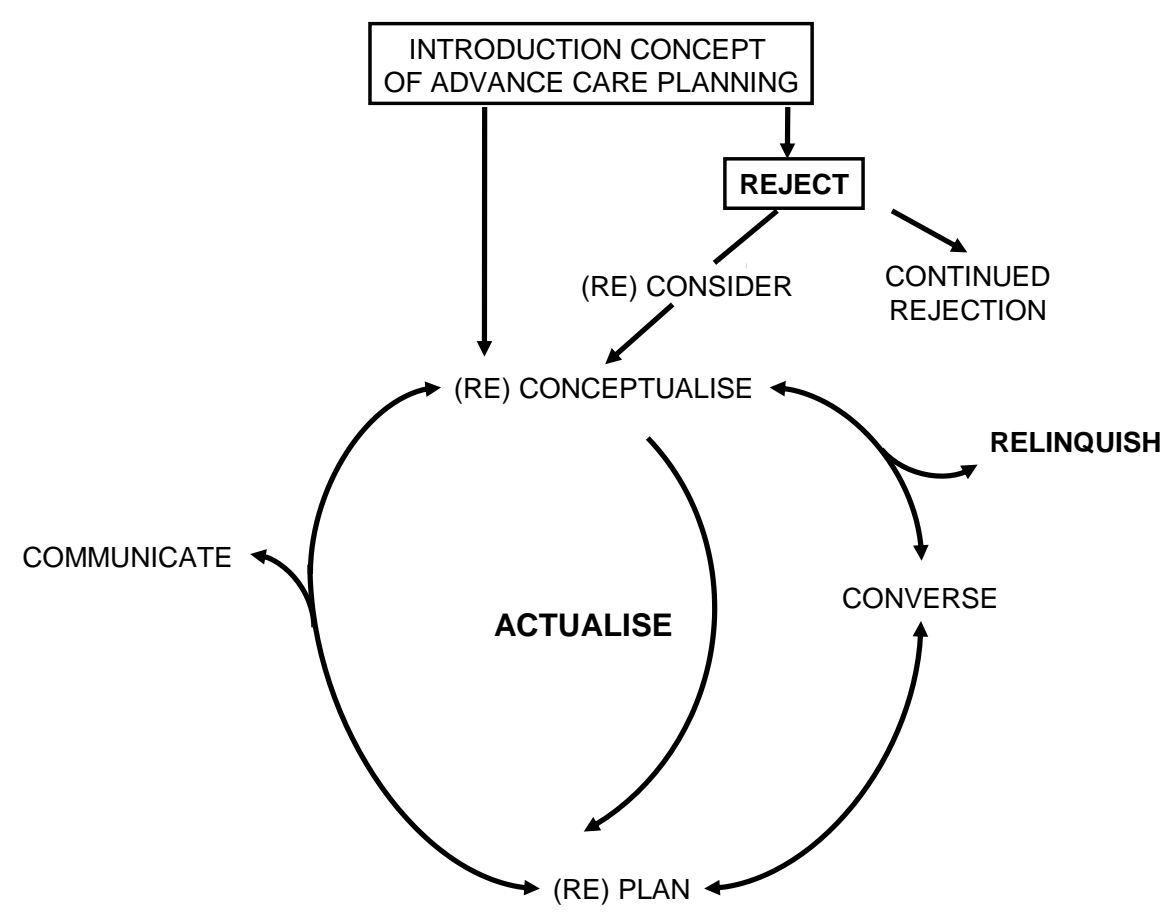




\section{Appendix 1 Information on Case Scenarios for Patients}

It is important to note that these scenarios are examples of imaginary cancer patients. They are not intended to represent your own condition or how your own illness may progress, though there may be similarities. Some people are comfortable talking about the issues that arise in the scenarios that you will be reading. It is also possible that these examples may make you feel uncomfortable. If so, please inform the researcher immediately and we can end the interview or provide support. You will be offered the opportunity to ask questions or obtain further support at the end of the interview.

\section{Case scenario 1}

You have been diagnosed with cancer in the last few weeks. At this stage, the cancer is limited to one area and has not spread. The diagnosis comes as a great shock to you and your family. You still feel well and are able to perform all your normal activities. You can think clearly and are able to continue working. Family and friends tell you that it is hard to believe that you have been diagnosed with cancer. You are waiting to be seen by a cancer specialist to discuss options for treatment of your cancer. You are planning to proceed with any treatment that is offered to you.

\section{Case scenario 2}

You have cancer that has spread from its original site. You have seen a specialist and have had one serious infection related to your chemotherapy treatment. You become very unwell and require admission to an intensive care unit. The doctors may plan surgery to remove the tumour if you show a positive response to chemotherapy. They explain that there are some risks associated with having surgery. This may include time spent in an intensive care unit under sedation.

You feel tired following chemotherapy but otherwise feel reasonably well.

\section{Case scenario 3}

You have cancer that has spread to your liver and areas of your brain. You have completed standard chemotherapy and have now been offered a drug on a clinical trial. The oncologist has explained that this is experimental treatment. You occasionally feel confused and need some help with dressing and feeding yourself. The doctors are going to give you some radiotherapy to your brain and have said there is a slight chance of improvement in your condition. You are able enjoy time with your family and friends but get tired easily and have given up your job. You have no pain but have lost weight. 


\section{Case scenario 4}

Your cancer is now very advanced. You have completed all available treatment to try and control the cancer and now spend increasing amounts of time in bed. You have pain that is controlled with regular medication. You sometimes feel sick or short of breath and also require medication for this. You are now eating and drinking very small amounts. The doctors have told you that you are very unwell. You have friends and family who are very supportive. 